\title{
Research on Rheological Disturbance Damage Laws of Rock in the Strength Limit Neighborhood
}

\author{
Changliang Lu*, Bo Wang, Shiyu Hu, Zikang Huang \\ School of Safety Engineering, North China Institute of Science and Technology, Beijing 101601, China \\ Email: 595605675@qq.com
}

\begin{abstract}
As coal mining enters deep, the rock is close to the strength limit neighborhood, and it is extremely susceptible to deformation and damage due to disturbance. In order to study the damage evolution law of rock in the strength limit neighborhood, red sandstone is selected as the research object, and the self-developed RRTS-IV rheological disturbance experimental system and nuclear magnetic resonance test equipment are used to carry out the gradual loading test of the rock rheological disturbance effect. Through the analysis of the test results, it is concluded that the instantaneous elastic modulus damage of the rock is accelerated rapidly with the increase of the number of disturbances in the strength limit neighborhood, and the disturbance becomes proportional to the instantaneous strain of the rock. At the same time, through parameter identification of the rock rheological data under different disturbance times, the rheological damage constitutive model is established, and the test results are in good agreement with the fitted curve. This shows that the establishment of this model is reasonable, and it can reflect the damage characteristics of the rock in the strength limit neighborhood under the action of different disturbance times, and the relaxation time and the disturbance times increase exponentially.
\end{abstract}

Keywords: strength limit neighborhood, constitutive relationship, relaxation time, rheological disturbance

\section{Introduction}

With the increase of coal mining depth, the surrounding rock of the roadway with larger buried depth gradually approaches the strength limit range. At this time, a small disturbance in the roadway will cause cracks in the surrounding rock, resulting in changes in the mechanical properties of the surrounding rock, and then instability and destruction of the roadway. Therefore, studying the damage evolution law and the damage constitutive relationship of the rock under the rheological disturbance has important guiding significance for the excavation and support of the roadway.

With the active construction of various underground projects and the in-depth understanding of various rock materials and engineering properties, important progress has been made in the timeliness and rheological properties of rocks. At the same time, there are many studies on rock rheology and rheological disturbances. Professor Gao Yanfa proposed the "rock creep disturbance effect" ${ }^{[1-2]}$, and carried out related tests to obtain the limit neighborhood range of rock strength. Wang Bo, Gao Changyan et al. ${ }^{[3-6]}$ used self-developed test equipment to conduct rheological disturbance tests on red sandstone. Through the analysis of rheological test results, they obtained the limit neighborhood range of the rock uniaxial and triaxial rheological disturbance intensity, and established a rheological constitutive model. Many scholars have also conducted a lot of research on the constitutive model of rock rheological damage and microscopic damage monitoring. Yang Chunhe ${ }^{[7]}$ used Xie Heping ${ }^{[8]}$ experimental research foundation to analyze the creep change law of salt rock and established a nonlinear creep constitutive model. Xu Weiya ${ }^{[9]}$ analyzed the creep law of green schist through triaxial test, used the Bingham model to analyze the creep attenuation, introduced a nonlinear function in the uniform speed stage, and introduced damage in the accelerated creep stage, and established the creep damage evolution. Construction model. Jiang Yuzhou ${ }^{[10]}$ et al. described the three stages of creep based on the nonlinear hardening function and damage evolution characteristic equation, combined with Maxuell's creep model. This model is not easy to determine during the creep acceleration stage. Chen ${ }^{[11]}$ used the mechanical properties of damage evolution to analyze the creep damage evolution characteristics of salt rock, and established the salt rock failure strength criterion. Wang Wei et al. ${ }^{[12]}$ based on Burgers, established the rheological damage constitutive equation by analyzing the indoor rheological test of red sandstone, and carried out parameter identification to verify the accuracy of the model. Combined with actual engineering, the calculation results showed that the calculation results were more accurate. Wang Laigui et al. ${ }^{[2]}$ analyzed the results of rock creep, performed parameter inversion, and established an accelerated creep expression equation. At present, there are in-depth researches on the macroscopic failure test of rock uniaxial and triaxial rheological disturbance, as well as the establishment of rock uniaxial and triaxial creep damage model. However, there are few studies on the change law of rock rheological 
damage and the establishment of constitutive relationship under disturbance, especially the research on the constitutive relationship of rock damage caused by rheological disturbance in the "strength limit neighborhood".

In this paper, red sandstone is selected as the research object, and the rock rheological test device is used to determine the long-term strength of the rock; then the rock specimen is loaded to the stress threshold selected by the "strength limit neighborhood range" to perform corresponding rheological changes. Perturbation frequency impact perturbation; analysis of the influence of disturbance on the rheological damage characteristics of red sandstone; combined with the Nishihara rheological model to establish a constitutive model for the lower rheological loss of disturbance, and use the LM (Levenberg-Marquardt) algorithm to identify the disturbance curve to verify the constitutive model Accuracy.

\section{Test overview and determination of strength limit neighborhood}

\subsection{Determination of the range of the strength limit neighborhood}

The test uses the independently developed RRTS-IV rheological disturbance experimental system. As shown in Figure 1 , the test device includes an impact disturbance device and a dynamic test analyzer. The rock specimens were drilled and cut into cylindrical specimens of diameter $\times$ height $(25 \times 50 \mathrm{~mm})$, and rock specimens with similar porosity and pore size distribution were selected by nuclear magnetic resonance technology for testing.

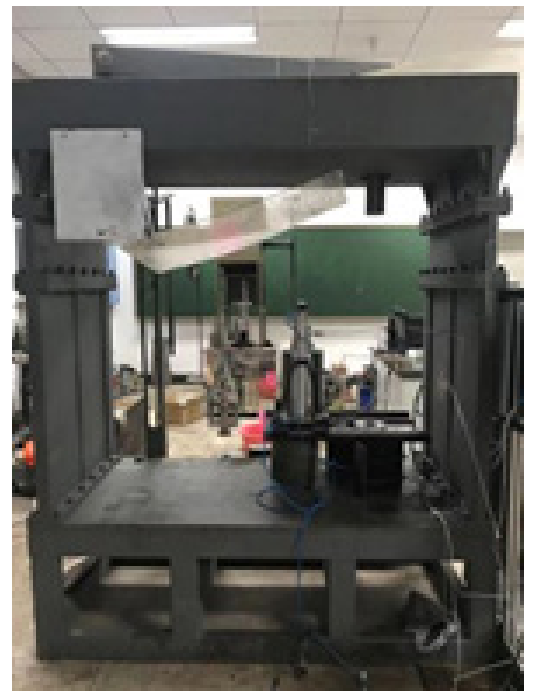

Figure 1. RRTS- IV rock creep disturbance effect test system

Before the test, two specimens were selected for the conventional uniaxial compression test on the RMT testing machine to provide the basis for the uniaxial rheological disturbance test. The numbers of the two test pieces are L-1 and L-2 respectively. The compression test data is shown in Table 1. The mean value of the uniaxial strength ultimate stress (uniaxial compressive strength ) is $63.80 \mathrm{MPa}$, and the mean value of the axial strain is $672 \times 10-6 \mu \varepsilon$. The transverse strain splitting surface is in contact with the probe, resulting in a large difference in the data, so the data is not taken as a follow-up study.

Table 1. Uniaxial compression test data

\begin{tabular}{cccccc}
\hline Specimen number & Height/mm & Diameter/mm & Strength limit/MPa & Axial strain/10-6 & Transverse strain/10-6 \\
\hline L-1 & 50.42 & 25.34 & 63.03 & 6648 & 3812 \\
L-2 & 50.44 & 25.17 & 64.57 & 6801 & 9647 \\
\hline
\end{tabular}

Two specimens (A-1, A-2) are selected for the rock rheological test, and the uniaxial compression test data is obtained. The component loads of the two specimens are 26, 36, 46, and $56 \mathrm{MPa}$. Figure 2 is a graph of rock rheological changes. 


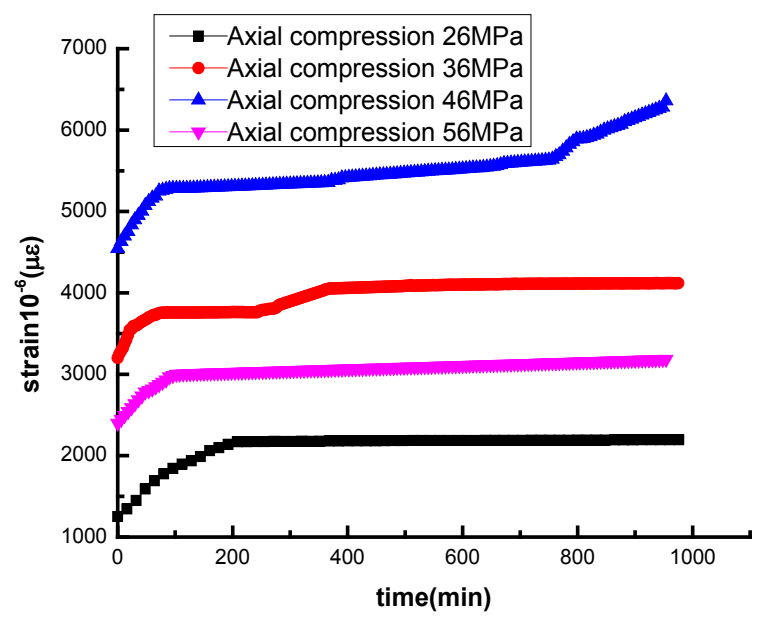

Figure 2. Rheological test curve of rock

It can be seen from the above Figure 2 that under the action of the first two levels of load, the rock strain rate remains basically unchanged. Under the third-level load, the rock deformation continued to increase in the first 400 minutes, indicating that under the condition of a relatively small axial stress level, the rock damage is mainly due to the closure of the original cracks and the compaction of internal pores. Rheology tends to be stable, and its manifestation is mainly deceleration ${ }^{[13]}$. When the fourth-level load is applied, the rock strain suddenly increases with time, the rheological rate is greater than zero, the rock tends to be in an unstable state, and the rock enters an accelerated rheological state. Wang Bo, Gao Changyan et al. ${ }^{[14]}$ determined the long-term strength range of the rock through the change of the rock stressstrain curve. The long-term strength interval of the rock is $46 \sim 56 \mathrm{MPa}$, and the average value of the long-term strength can be 51.0MPa. The long-term strength of the rock determines its strength limit neighborhood range. First, determine the maximum uniaxial pressure value of the rock (the uniaxial compressive value of the rock ), determine the longterm strength value of the rock by analyzing the rheological curve, and then determine the range of its strength limit neighborhood threshold ${ }^{[1]}$. Determine the width of the left neighborhood, that is, the left boundary stress of the strength limit neighborhood of the soft rock. The above tests have determined the uniaxial average compressive strength of the rock and the long-term strength of the rock $(63.80 \mathrm{MPa}, 51.0 \mathrm{MPa})$, so the left boundary stress of the rock strength limit neighborhood is $51.0 \mathrm{MPa}$. This test selects the right boundary stress (and the axial stress 52MPa) where the rock is in the vicinity of the strength limit.

\subsection{Test overview}

The test selects 6 specimens, through the above-mentioned long-term strength analysis of the rock, the test process is as follows.

(1) The selected 6 test pieces were subjected to weighting, and they were loaded to 52MPa for rheological test. After the rheology is relatively stable, impulse disturbance is applied. There is a 15 -second difference in the time interval between the disturbances before and after. Record the instantaneous strain change value of the rock immediately after the action of different disturbance times. The rock specimen is shown in Figure 3.

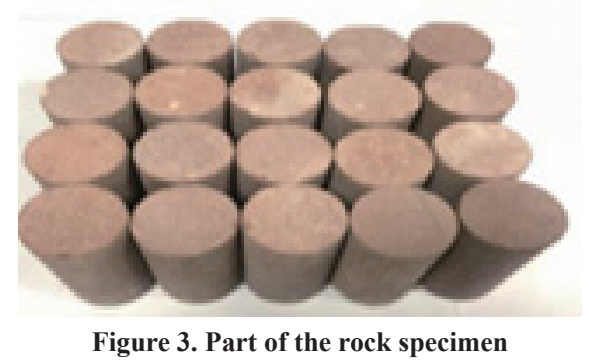

(2) The impact disturbance energy applied by each test piece is expressed by $\Delta \mathrm{W}$, the mass of the disturbance ring is $\mathrm{M}(2.5 \mathrm{Kg})$, the impact drop height is $\mathrm{H}$, and $8 \mathrm{~cm}$ is selected (Figure 4). Under the premise of ignoring other frequency effects and neglecting energy loss, the expression of energy acting on the specimen is: 


$$
\Delta W=\frac{M \sqrt{2 g h}}{A}
$$

A is the area of the bottom surface of the cylindrical specimen; $g$ is the acceleration due to gravity, taking $g=9.8 \mathrm{~m} / \mathrm{s} 2$.

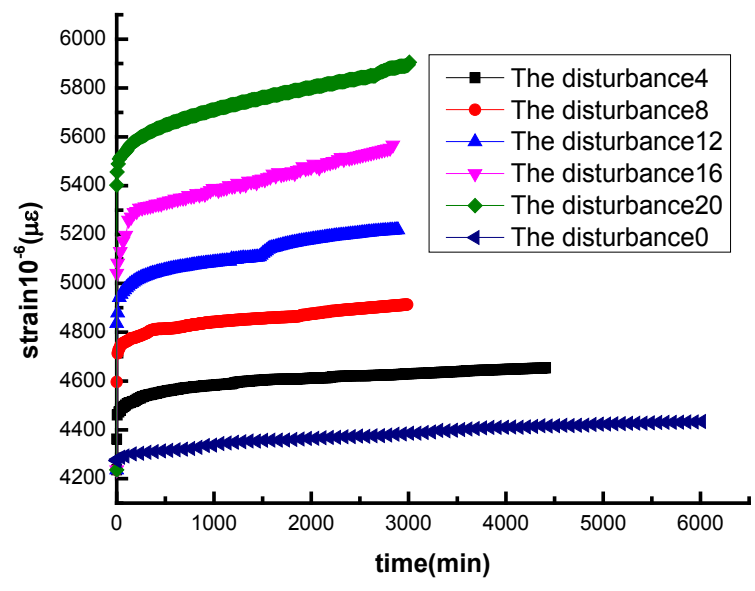

Figure 4. Rheological curve under the disturbance

Using the nuclear magnetic resonance technology (NMR) developed by Suzhou Newmai Company, the rock specimens that are disturbed by impact can be visually observed the changes in the pore size and pores of the rock. The nuclear magnetic resonance technique (NMR) is shown in Figure 5.



Figure 5. NMR system



Figure 6. Instantaneous strain of red sandstone under different numbers of disturbance

According to Figure 6, when the rock is in the neighborhood of the strength limit, the instantaneous strain is proportional to the number of disturbances. This shows that the instantaneous elastic modulus decreases with the increase of the number of disturbances, and the increase of the number of disturbances roughly accelerates the damage of the instantaneous elastic modulus of rock. 


\section{The relationship between rock rheological disturbance effect and damage constitutive}

\subsection{Rock damage evolution equation}

The rock elastic modulus is used to express the damage change. The relationship between the instantaneous damage changes before and after the rock disturbance is as follows ${ }^{[15]}$.

$$
D_{S}=1-\frac{E_{1}}{E_{0}}
$$

In the formula, E0 is the elastic modulus of the rock before the disturbance, and E1 is the instantaneous elastic modulus of the rock after the disturbance.

Under the condition of unidirectional compression, the rock is expressed as follows according to Kachnow's rheological damage law:

$$
\dot{D}=A \sigma(1-D)^{-v}
$$

In the formula: $\mathrm{A}$ and are the material constants related to the disturbance, and $\mathrm{D}$ is the disturbance damage factor; the test results under the action of different disturbance times are analyzed, and the law of its change with the disturbance is determined.

Assuming that the rheological damage is $\mathrm{D}=0$ at $\mathrm{t}=0$, and $\mathrm{D}=1$ at the time of fracture, the damage evolution process of rock rheology with time can be obtained by formula (2):

$$
D=1-\left(1-t / t_{F}\right)^{1 /(1+v)}
$$

Among them:

$$
\begin{aligned}
& t_{F}=\left[A(1+v) \sigma^{v}\right]^{-1} \\
& \beta=1 /(1+v)
\end{aligned}
$$

$\mathrm{t}_{\mathrm{F}}$ is the rock rheological damage and fracture time, and is the rock damage evolution and failure characteristics.

The relationship between the damage change factor D and is shown in Figure 7. It can be seen from Figure 7 that the smaller the value of, the faster the change rate of the rock in the rheological acceleration stage, and its manifestation is mainly brittle failure. Conversely, the slower the rate of change in the rheological acceleration stage, the manifestation is ductile failure.

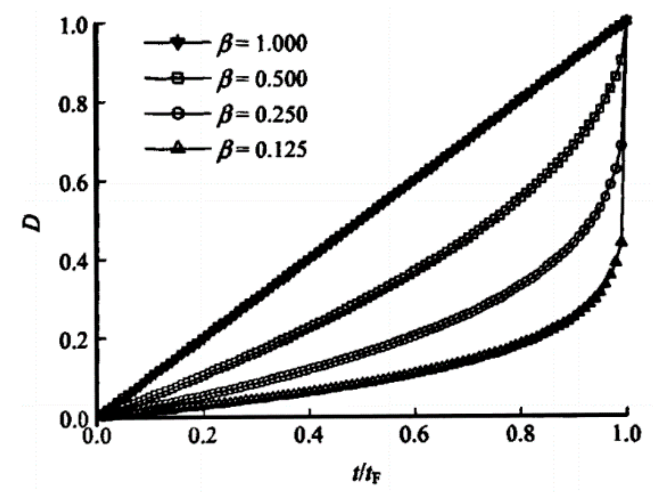

Figure 7. The relationship with $\mathbf{t} / \mathbf{t}_{\mathrm{F}}$

\subsection{Establishment of constitutive model of rheological damage under disturbance}

The three stages of rheology are the inherent rheological properties of the rock. Due to the different axial stresses applied to the rock, the deformation of the rock caused by the disturbance effect is different. When the axial pressure is low, the disturbance deformation is recoverable; on the contrary, the disturbance deformation cannot be recovered. Therefore, rock rheology can be divided into linear rheology and nonlinear rheology.

The Nishihara model is a combination of Hooke body $(H)$, viscoelastic body $(\mathrm{N} / \mathrm{H})$ and viscoplastic body $(\mathrm{N} / \mathrm{St}$. V) 
(Figure 8), because it can better reflect the elasticity-viscoelasticity of rocks. The viscoplastic deformation characteristics are widely recognized. When the rock is under unidirectional stress, its rheological equation can be expressed as follows ${ }^{[16]}$ :



Figure 8. Xiyuan model

(1) When the rock is under unidirectional stress, and when .

At this time, it will be used as a rigid body structure. The model has the same characteristics as the generalized Kelvin style, and its constitutive equation is as follows:

$$
\varepsilon=\left(\frac{1}{E_{B}}+\frac{1}{E_{K}}\right) \sigma_{C}-\frac{1}{E_{K}} \sigma_{C} e^{\frac{E_{K}}{\eta_{K}} t}
$$

It can be seen from the formula that when stress, the rheological deformation is recoverable and in a steady-state rheological state. At $\mathrm{t}=0$, the generalized Kelvin body has no effect. As time changes, the viscous damping cylinder will also have no effect. So in the end, it is only the series connection of EK and EB; at the same time, relaxation will not make the stress zero.

(2) When .

This model is similar to the Burgers model, except that is replaced with, and its constitutive equation is:

$$
\varepsilon=\frac{\sigma_{c}-\sigma_{s}}{\eta_{B}} t+\frac{\sigma_{c}}{E_{B}}+\frac{\sigma_{c}}{E_{K}}\left(1-e^{\frac{E_{K}}{\eta_{K}} t}\right)
$$

It can be seen from the formula that when $\mathrm{t}=0$, only the spring is effective, and shows a metastable rheology with time ${ }^{[15]}$.

In the formula: is the long-term strength of the rock, and EB and EK are the elastic modulus of the Nishihara model respectively.

In order to express more clearly the rheological process of rock under the action of different disturbance times, the above equations (1) and (3) are introduced into (6) and (7). The rheological disturbance damage constitutive model equation is as follows:

$$
\varepsilon= \begin{cases}\frac{\sigma_{C}}{E_{B}\left(1-D_{S}\right)}+\frac{\sigma_{C}}{E_{K}(1-D)}\left(1-e^{-E_{K} t / \eta_{K}}\right) & \left(\sigma<\sigma_{S}\right) \\ \frac{\sigma_{C}}{E_{B}\left(1-D_{S}\right)}+\frac{\sigma_{C}}{E_{K}(1-D)}\left(1-e^{-E_{K} t / \eta_{K}}\right)+\frac{\left(\sigma_{C}-\sigma_{S}\right) t}{\eta_{B}(1-D)} & \left(\sigma<\sigma_{S}\right)\end{cases}
$$

From the above formula (8), it can be seen that the disturbance has an effect on the instantaneous elastic modulus of the rock, and the follow-up effect of the disturbance exists in the entire rheological damage evolution. This is consistent with the previous description of rheological damage. The above formula (8) can better reflect the deceleration, uniform velocity and acceleration stages of rock rheology, and the establishment of this model is more reasonable.

\section{Validation and analysis of constitutive parameters}

According to the rheological disturbance curve of the rock entering the neighborhood of the strength limit, the correctness of the establishment of the model is verified.

The instantaneous elastic modulus is calculated under the action of different disturbance times of the red sandstone, as shown in Table 2. Assuming that when there is no disturbance, the damage is 0 , then the damage variation curve caused by the disturbance is shown in the figure below. Using the exponential function in mathematics to fit it, the following expression equation is obtained:

$$
D_{S}=-0.0598+0.0646 e^{0,0843 s}
$$


Table 2. Instantaneous elastic modulus and disturbance energy change

\begin{tabular}{cccccc}
\hline Number of disturbance/time & E/GPa & Ds & Number of disturbance/time & E/GPa & Ds \\
\hline 0 & 12.27 & 0 & 12 & 10.70 & 0.12388 \\
4 & 11.92 & 0.02892 & 16 & 10.27 & 0.17301 \\
8 & 11.31 & 0.07832 & 20 & 9.62 & 0.29584 \\
\hline
\end{tabular}

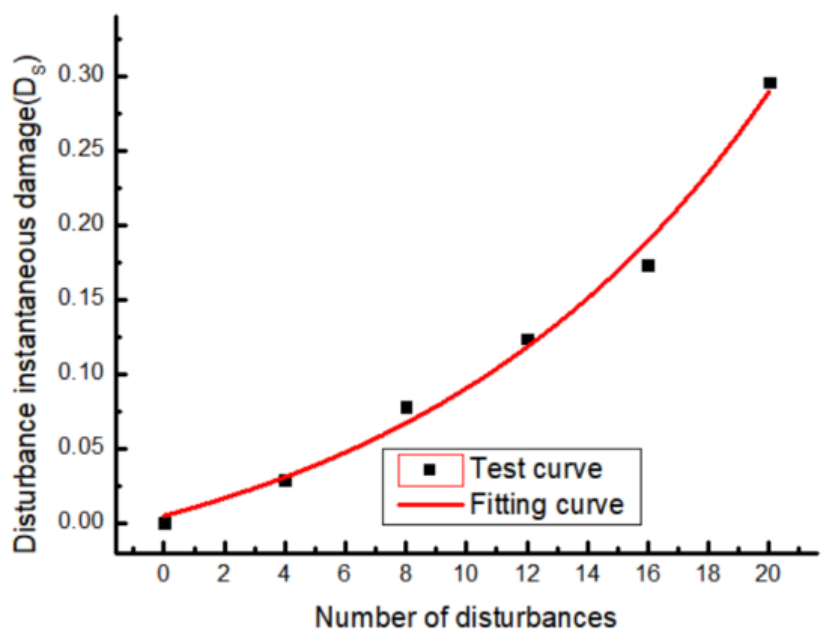

Figure 9. Relation between instantaneous damage change and the number of disturbance

It can be seen from Figure 9 that ( $\mathrm{S}$ is the number of disturbances) the fitted curve is in good agreement with the test results, indicating that Equation 9 is reasonable.

Combine equations (3) and (7). Assuming that $\mathrm{t}_{\mathrm{F}}=P_{1}, \beta=P_{2}, E_{2}=P_{3}, 1 / \tau=P_{4}(\tau$ is the relaxation time), the fitting is performed by the 1stOpt nonlinear least squares method based on the LM algorithm. The advantage of this method is that it does not need to select the initial value problem, can accurately identify the parameters of the curve under rheological disturbance, and obtain the red sandstone model parameters under the action of different disturbance times (Table 3). Comparing the fitted curve with the test curve (as shown in Figure 10) shows that the curve fits well. This shows that the establishment of the rheological damage model is reasonable and can accurately reflect the rheological damage characteristics of red sandstone under different disturbance times.

Table 3. Rheological model parameters

\begin{tabular}{ccccc}
\hline Number of disturbance/time & P1/h & P2 & P3/MPa & 1537 \\
\hline 0 & 63.7 & 0.0850 & 941 & 0.0357 \\
4 & 45.2 & 0.1732 & 826 & 0.2237 \\
8 & 32.1 & 0.3624 & 618 & 0.4108 \\
12 & 26.3 & 0.4571 & 442 & 0.6620 \\
16 & 19.6 & 0.7228 & 328 & 0.8363
\end{tabular}




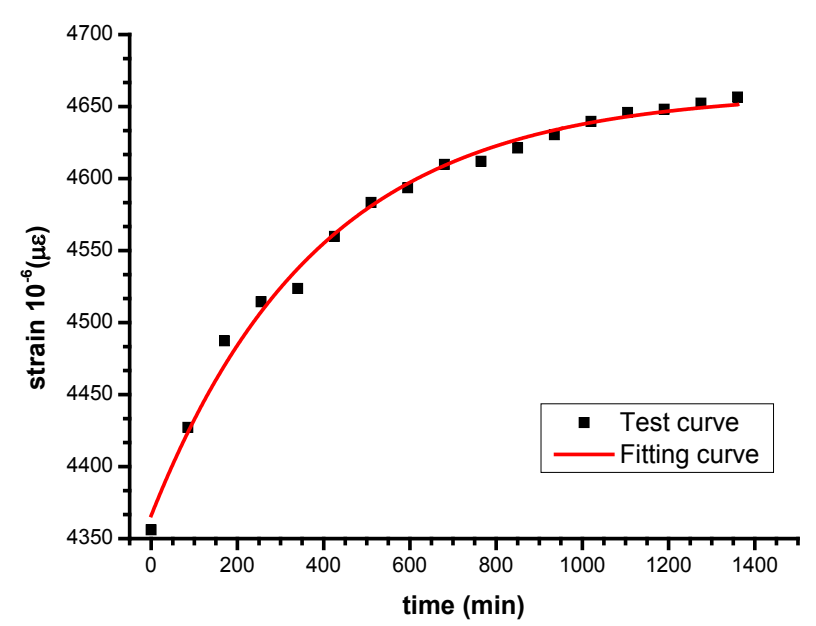

(a) Number of disturbance: 0

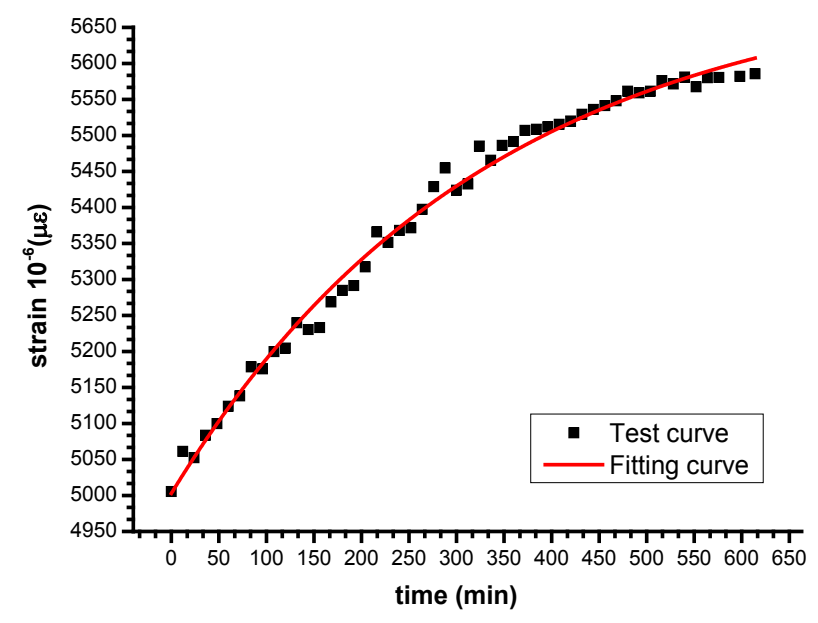

(c) Number of disturbance: 8

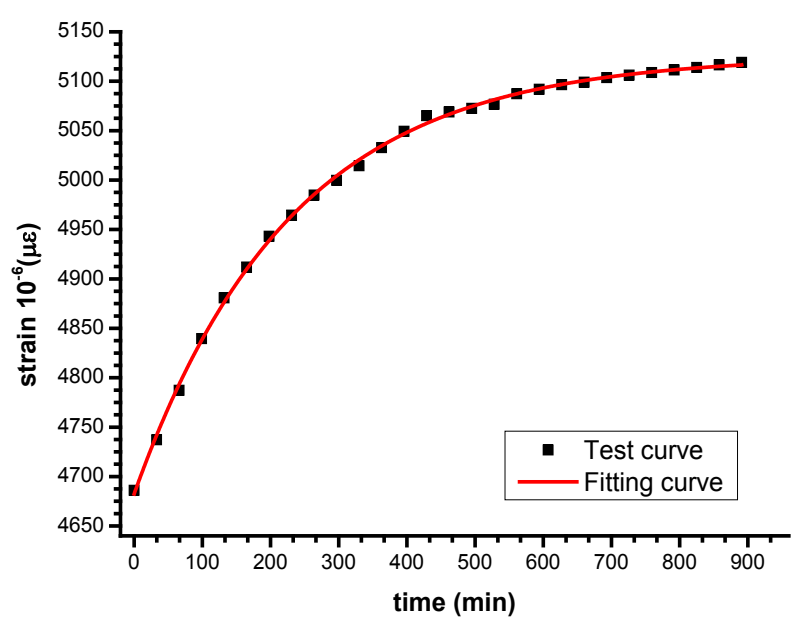

(b) Number of disturbance: 4



(d) Number of disturbance: 12

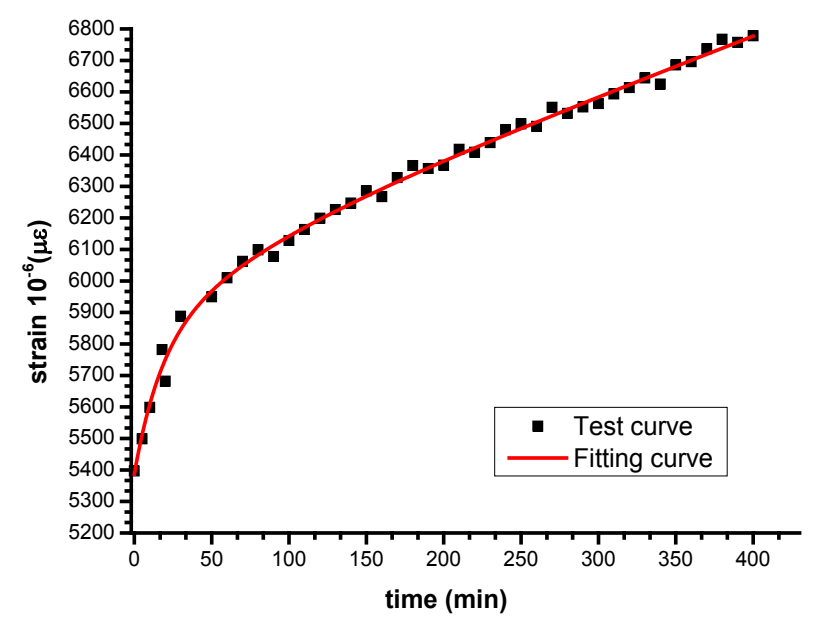

(e) Number of disturbance: 16

Figure 10. Comparison of test results and fitting curves under different numbers of disturbance

According to the results of the red sandstone rheological disturbance damage constitutive model parameter identification (Table 3), the damage evolution law of the rock under different disturbance times in the "strength limit neighborhood" is analyzed. 
Analyze the relationship between rock damage and failure time $t_{\mathrm{F}}$ under different disturbance times. It can be seen from Figure 11 that when the number of disturbances increases, the damage and failure time $\mathrm{tF}$ of the rock decreases greatly, and the exponential function is used for fitting, and the relationship expression equation is established:

$$
t_{F}=-4.398+67.31 e^{-S / 14.13}\left(\mathrm{R}_{2}=0.9835\right)
$$

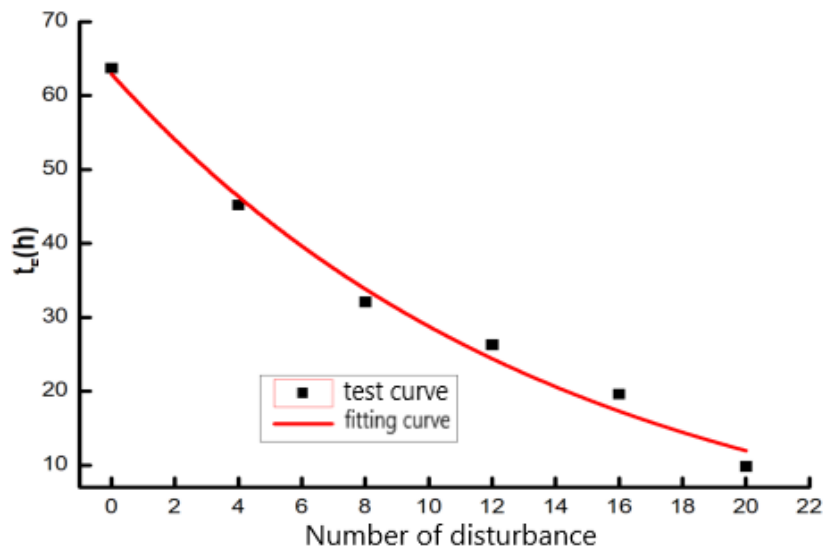

Figure 11. Variation of rheological damage time of red sandstone with the number of disturbance

\section{Conclusion}

(1) The perturbation effect in the neighborhood of the strength limit is extremely sensitive to rock damage, and the damage is proportional to the number of perturbations. The increase in the number of disturbances causes the instantaneous elastic modulus damage of the rock to accelerate rapidly.

(2) Through the parameter identification of the rock rheological data under different disturbance times, the rheological constitutive model is established, and the 1 stOpt is used for fitting comparison, and the results of the disturbance test in the strength limit neighborhood are in good agreement with the fitting curve. . This shows that the establishment of this model is reasonable and can reflect the damage characteristics of the rock under the action of different disturbance times when the rock is in the neighborhood of the strength limit.

\section{Acknowledgments}

This paper is supported by Natural Science Foundation of Hebei Province (E2019508088) and Fundamental Research Funds for Central Universities (3142020062).

\section{References}

[1] Gao Yanfa, Fan Qingzhong, Cui Xihai, etc. Experimental study on rock rheology and its disturbance effect. Beijing: Science Press; 2007.

[2] Gao Yanfa, Xiao Huaqiang, Wang Bo et al. Research on rock rheological disturbance effect and its constitutive relationship. Chinese Journal of Rock Mechanics and Engineering. 2008; 27(S1): 3180-3185.

[3] Wang Bo, Gao Changyan, Chen Xuexue, et al. Uniaxial compression test study on rock rheological disturbance characteristics. Journal of China Coal Society. 2017; 42(6).

[4] Wang Bo, Gao Changyan, Chen Xuexue, Liu Chongyang Rock Rheological Disturbance Effect Triaxial Compression Test Research. Journal of China Coal Society. 2018; 43(S2): 403-411.

[5] Wang Bo, Liu Chongyang, Gao Changyan, Yan Gang, Yang Jianlin, Gu Changwan. Experimental study on rock uniaxial creep disturbance in the neighborhood of strength limit. Coal Science and Technology. 2018; 46(07): 81-86.

[6] Wang Bo, Gao Changyan, Chen Xuexue, Liu Chongyang Rock Rheological Disturbance Effect Triaxial Test System. Journal of China Coal Society. 2018; 43(02): 433-440.

[7] Yang Chunhe. Chen Feng, Zeng Yijin. Research on the relationship of salt rock creep damage. Chinese Journal of Rock Mechanics and Engineering. 2002; 21(11).

[8] Xie Heping. Damage mechanics of rock concrete. Xuzhou: China University of Mining and Technology Press; 1990.

[9] Xu Weiya, Zhou Jiawen, Yang Shengqi, et al. Study on the constitutive relationship of green schist creep damage. 
Chinese Journal of Rock Mechanics and Engineering. 2006; 25(1): 3093-3097.

[10] Jiang Yuzhou, Xu Weiya, Wang Ruihong, et al. Research on nonlinear creep damage model of rock. Journal of China University of Mining and Technology. 2009; 38(3): 331-335.

[11] Wang Wei, Lu Jun, Wang Haicheng, et al. Research on sandstone rheological damage model and its engineering application. Chinese Journal of Rock Mechanics and Engineering. 2012; 31(S2): 3650-3658.

[12] Wang Laigui, He Feng, Liu Xiangfeng, et al. Nonlinear creep model and stability analysis of rock specimens. Chinese Journal of Rock Mechanics and Engineering. 2004; 23(10).

[13] QIN Yuepeng, WANG Lin, SUN Wenbiao, et al. Study on rheological theory model of rock damage. Chinese Journal of Rock Mechanics and Engineering. 2002; 21(Supp.2): 2291-2295.

[14] Yu Yongjiang, Liu Feng, Zhang Wei, et al. Rheological disturbance effect experiment and constitutive model study of water rich soft rock. Vibration and impact. 2019; 38(12): 201-205.

[15] Song Yijiang. (2013) Study on creep mechanical properties and constitutive model of deep fractured surrounding rock. Ph.D. Thesis, China University of Mining and Technology, Xuzhou.

[16] Tao Bo, Wu Faquan, Guo gaimei, Zhou Ruiguang. Adaptability of Xiyuan model to rheological properties of rock and determination of its parameters. Chinese Journal of Rock Mechanics and Engineering. 2005; (17): 3165-3171. 